\title{
First-line Therapeutic Strategies for Myelodysplastic Syndromes
}

\author{
Valeria Santini
}

\begin{abstract}
The precise diagnostic tests and subsequent prognostic stratification for patients with myelodysplastic syndrome (MDS) are often cumbersome, yet they are the basis of successful therapy. Diverse treatment options are available for these patients; however, the decisions in real-life are often not grounded on the available evidence. Although the International Prognostic Scoring System and revised International Prognostic Scoring System are still driving the medical approach to MDS patients, additional variables must be considered when therapeutic intervention is needed. A rational scheme for first-line therapy is described that allows for the possibility of selecting the optimal individual therapy for MDS patients.
\end{abstract}

Clinical Lymphoma, Myeloma \& Leukemia, Vol. 17, No. S1, S31-6 (c) 2017 Elsevier Inc. All rights reserved. Keywords: Erythropoietic stimulating agents, First-line therapy, Hypomethylating agents, Myelodysplastic syndromes, Somatic mutations

\section{Introduction}

Myelodysplastic syndrome (MDS) refers to a heterogeneous group of diseases, and it is quite difficult at present to continue to refer to them as a unique nosologic entity. The diagnosis is difficult and requires expertise in morphology, cytogenetics, and, increasingly, molecular techniques. The prognosis is dominated by the disease characteristics; however, individual patient-related variables such as age, frailty, comorbidities, and personal wishes and compliance could also be determinants in the choice of therapy. Regardless, it is clear that the first step to establishing a good treatment strategy is to properly evaluate each suspect case of MDS and, once the diagnosis has been confirmed, provide an accurate prognostication. The International Prognostic Scoring System $\left(\text { IPSS) }{ }^{1} \text { and the revised IPSS (IPSS-R) }\right)^{2}$ are tools widely used in first evaluations. Both scoring systems have taught us to consider MDS patients in terms of having a low or a high risk of progression to acute leukemia and to consider the therapies accordingly. Nevertheless, the depth of cytopenia in "low-risk" patients could constitute an obstacle to maintaining a decent quality of life and could result in death, in the absence of any disease progression. In contrast, patients with "high-risk" MDS could experience prolonged

MDS Unit, Department of Hematology, Azienda Ospedaliera Universitaria Careggi, University of Florence, Florence, Italy

Submitted: Feb 28, 2017; Accepted: Feb 28, 2017

Address for correspondence: Valeria Santini, MD, Ematologia, MDS Unit, Department of Hematology, Azienda Ospedaliera Universitaria Careggi, University of

Florence, Largo Brambilla 3, Florence 50141, Italy

E-mail contact: santini@unifi.it survival with disease stabilization and an acceptable quality of life. At present, it is fundamental to determine the correct strategy to manage MDS, because, although several therapeutic options are possible, their sequence and, in particular, the first-line choice can be critical to the disease course.

First, the inception of therapy should be determined exclusively by the symptoms to actively alleviate them and on the possibility of delaying the progression to leukemia and eradicating the disease.

\section{First-line Treatment of Lower Risk MDS}

The management of lower risk MDS (ie, very low, low, and intermediate IPSS-R risk), as indicated by the most recent guidelines (National Comprehensive Cancer Network), ${ }^{3}$ has recently been revisited. ${ }^{4}$ The presented algorithm was created from quite articulated evidence, and each drug option was determined from several individual- and disease-related parameters. The recommendations include first and subsequent lines of treatment. The focus of the present report was the choice of first-line therapy (Figure 1).

Symptomatic anemia is the most frequent trigger for therapeutic intervention. As much as possible, transfusions should be used only in emergency situations. Whenever possible, transfusion should not be considered as standard continuous treatment without testing alternative approaches, both to maintain the best quality of life and to avoid cardiac and systemic complications. ${ }^{4}$

When red blood cell (RBC) transfusions are necessary, and they can be for most MDS patients at some stage of the disease, they should be given using a hemoglobin threshold derived from individual symptoms, not from the routine use of transfusions in other 


\section{First-line Therapy for MDS}

\section{Figure 1 First-line Therapeutic Algorithm for Lower Risk Myelodysplastic Syndrome (MDS)}

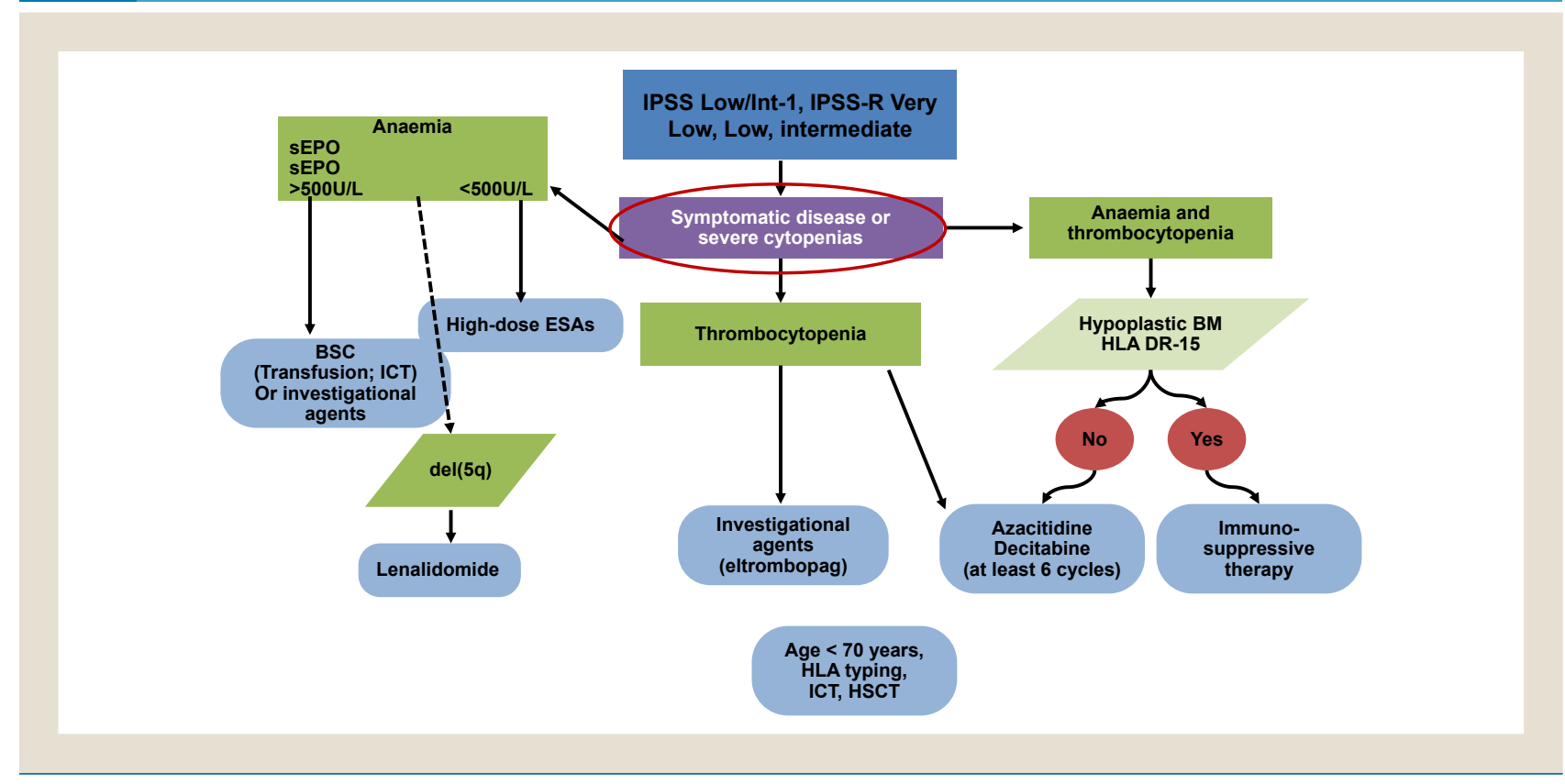

Abbreviations: ATG = antithymocyte globulin; BM = bone marrow; BSC = best supportive care; $E S A=$ erythropoietic-stimulating agent; HLA = human leukocyte antigen; HSCT = hemopoietic stem cell transplantation; ICT = iron chelation therapy; SEPO = serum erythropoietin.

settings, which have a threshold of $8 \mathrm{~g} / \mathrm{dL}$. Nilsson-Ehle et $\mathrm{al}^{5}$ reported that good correction of anemia with transfusions is possible and that their efficacy in maintaining high hemoglobin levels correlated with quality of life in an equivalent manner to that of erythropoietic-stimulating agents (ESAs). However, the chronic RBC transfusions typically needed for patients with MDS cannot resolve chronic anemia. Although life-saving, such transfusions will not correct the morbidity and poor quality of life, because the transfusions will not usually normalize the hemoglobin levels. In addition, the transfusions expose the patients to fluctuating hemoglobin levels. Also, iron overload due to RBC transfusions can be deleterious to organs such as the liver and heart and to hemopoiesis itself. Thus, iron chelation therapy is recommended as a part of best supportive care, when $\geq 20 \mathrm{U}$ of RBCs have been transfused. ${ }^{6}$

ESAs can be effective in resolving the anemia of MDS and should be as first-line treatment. When feasible, ESAs should be used before transfusions (as defined by the International Working Group criteria $^{7}$ ) and, certainly, before the transfusion burden has become too great. Ideally, ESAs should be prescribed as soon as the hemoglobin levels significantly affect the patient's physical function. ESAs will achieve the best results in terms of erythroid response when used in IPSS lower risk MDS patients, with serum erythropoietin levels $<500 \mathrm{U} / \mathrm{L}$, without transfusion dependence, and with a normal karyotype and the absence of blasts in the bone marrow. ${ }^{8}$ The presence of pure erythroid dysplasia, low serum ferritin, very low and low IPSS risk, ${ }^{9}$ the presence of $<2$ somatic mutations, ${ }^{10}$ and the timely start of therapy, within 6 months of the diagnosis, will ensure the greatest rate of response. ${ }^{11}$ For such patients, the response has been $>70 \%{ }^{9}$ The optimal doses have been established as 30,000 to 80,000 U of erythropoietin (EPO; Epoetin alfa) ${ }^{12}$ and 150 to $300 \mu \mathrm{g}$ of darbepoetin alfa in subcutaneous injections weekly. ${ }^{13}$ Whether standard or higher doses of ESAs are preferable is still a matter of investigation. The preliminary results reported for 2 randomized registered trials comparing the safety and efficacy of EPO and darbepoetin alfa with placebo have further demonstrated the activity of ESAs in MDS, although at rates inferior to those published reported (EPO, $31.8 \%$ vs. placebo, $4.4 \%$; darbepoetin alfa, $14.7 \%$ vs. placebo, $0 \%) .{ }^{14,15}$ The lower rates mainly resulted from the interruption of ESA treatment in accordance with the study scheme, such that when the hemoglobin levels increased and approached $12 \mathrm{~g} / \mathrm{dL}$ ESA treatment was discontinued. However, the effect of ESAs on dysplastic erythropoiesis is temporary and disappears with interruption of ESAs.

A survival advantage has been suggested for patients receiving ESAs, ${ }^{16}$ although a recent comparison with an untreated matched population by our group indicated that this advantage is substantial only for a subgroup of MDS patients (Messa et al, manuscript submitted).

ESAs have no major contraindications for use in patients with MDS. No increase in thrombotic events and no hint of any increase in disease progression have been observed compared with non-ESA-treated patients, ${ }^{17}$ in contrast to reports of solid neoplasms. To maintain the response, the serum iron, vitamin $B_{12}$, and folate levels should be controlled. The most relevant difference with other hematologic and nonhematologic neoplasias is that the anemia of MDS is, by itself, "the" disease and is chronic and, therefore, requires continuous treatment. The response to ESAs is not immediate in MDS, although it is generally observed within 12 weeks. Thus, evaluations of the response before 12 weeks should not result in stopping the treatment and crossover to an alternative therapy. ${ }^{4}$

The addition of granulocyte colony-stimulating factor to ESAs has been reported to increase the rate of response. ${ }^{16}$ The use of 
granulocyte colony-stimulating factor combined with ESAs might, thus, be justified. However, its use as therapy for neutropenia has been not supported by any evidence of an advantage. Moreover, isolated neutropenia is infrequent, and patients with MDS have a good prognosis and low risk of developing acute myeloid leukemia. ${ }^{18}$ When neutropenia accompanies anemia and/or thrombocytopenia, more aggressive treatment is recommended, even for lower risk MDS, such as hypomethylating agents (HMAs) (see the next section).

Lower risk MDS patients with long-lasting RBC transfusion dependence or serum EPO $>500 \mathrm{U} / \mathrm{L}$ are not candidates to receive ESAs. However, for such patients, most available therapies are not effective. Recently, patients ineligible for ESA therapy were included in a randomized study of lenalidomide versus placebo. ${ }^{19}$ However, the results were quite disappointing. The overall response rate in this setting was only $8.6 \%$, placing on hold the use of lenalidomide as a first-line option for non-del5q patients. ${ }^{19}$

Although great number of MDS patients will have a response to ESAs, the response is not definitive, and the median response duration is approximately 2 years. For lower risk MDS with del(5q), the ESA response duration has been shorter and the rate of response overall more limited. ${ }^{20}$ A poor response to EPO can be attributed to high endogenous EPO levels at diagnosis in patients with $\operatorname{del}(5 q)$ MDS. In contrast, lenalidomide therapy, approved for MDS patients with transfusion-dependent, IPSS low-risk or intermediate-1 risk, del(5q) MDS, with or without additional cytogenetic abnormalities, is extremely effective ( $73 \%$ to $83 \%$ erythroid response, $56 \%$ transfusion independence). ${ }^{21}$ The $10-\mathrm{mg}$ dose resulted in a greater response than the $5-\mathrm{mg}$ dose $(61 \%$ vs. $49 \%$ transfusion independence) and greater cytogenetic response. ${ }^{21}$
It is a matter of debate whether $\operatorname{del}(5 q)$ MDS patients should receive lenalidomide only at the onset of transfusion dependence. One study (ClinicalTrials.gov identifier, NCT01243476; study of REVLIMID [lenalidomide] vs. placebo in patients with low risk myelodysplastic syndrome [SINTRA-REV]) compared lenalidomide and placebo for patients with newly diagnosed anemic del $(5 \mathrm{q})$ MDS. Because durable transfusion independence is associated with a significantly reduced risk of acute myeloid leukemia progression $(45 \% ; P=.022)$ and death $(51 \% ; P=.008)$, it would seem logical to delay the need for RBC transfusion by early treatment with lenalidomide.

The rate of a complete cytogenetic response after lenalidomide has been lower for patients with a TP53 mutation (0 of 7 with a TP53 mutation and 12 of 24 without; $\left.\chi^{2}, P=.024\right)$. Mutated TP53 will be present in approximately $19 \%$ of $\operatorname{del}(5 q)$ cases and predicts for a poor outcome and disease progression. ${ }^{22}$ In such cases, hematopoietic stem cell transplantation could be performed early; however, this therapy has also been associated with dismal outcomes. ${ }^{23}$ Patients diagnosed with $\operatorname{del}(5 \mathrm{q})$, irrespective of the complexity of karyotype, should be evaluated for the presence of a TP53 mutation. If TP53 is mutated, a more aggressive therapy strategy should be envisaged, although, at present, all available treatment options are less effective (with the possible exception of decitabine) in MDS patients with such a mutation. ${ }^{24}$

When anemia is not the sole severe and symptomatic form of cytopenia, HMAs, as an effective alternative first-line therapy, can be considered. However, HMAs have not been approved in the European Union for lower risk MDS. Their use should be restricted to lower risk MDS patients with $>1$ cytopenia to balance the risks and advantages of such chronic therapy. HMAs should also be

\section{Figure 2 First-line Therapeutic Algorithm for Higher Risk Myelodysplastic Syndrome (MDS)}

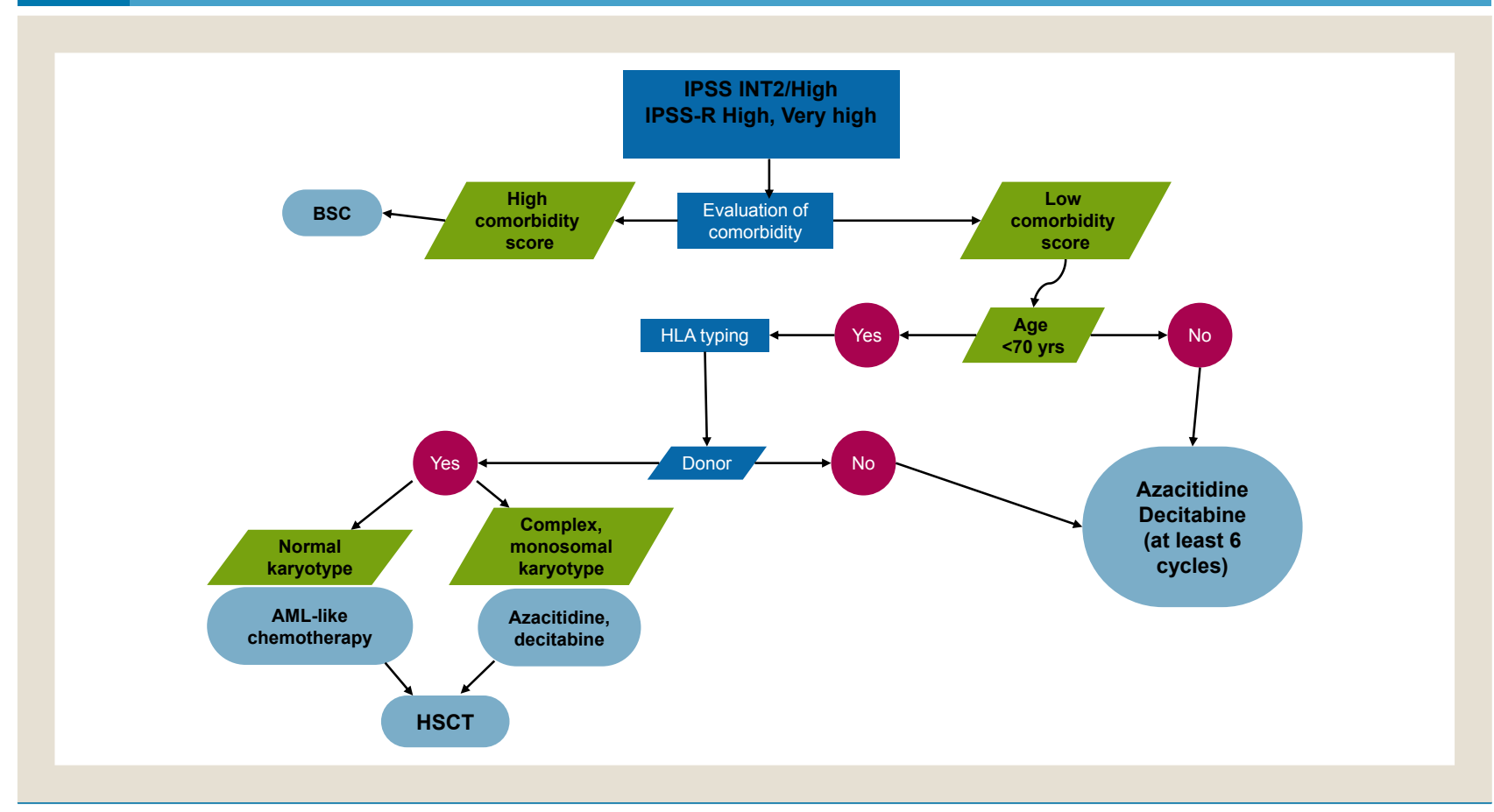

Abbreviations: $\mathrm{AML}=$ acute myeloid leukemia; $\mathrm{BSC}=$ best supportive care; HLA = human leukocyte antigen; HSCT = hematopoietic stem cell transplantation; IPSS INT2 = International Prognostic Scoring System intermediate risk 2. 
considered when a relatively young patient with lower risk MDS presents with somatic mutations with negative prognostic effects. For these patients, a more aggressive approach is needed, including transplantation. Both azacitidine and decitabine have shown efficacy in inducing transfusion independence and hematologic improvement in most treated patients and can also be an ideal bridge to transplantation for eligible patients. ${ }^{25,26}$

Standard-dose azacitidine $75 \mathrm{mg} / \mathrm{m}^{2}$ subcutaneously for 7 days every 28 days or 5 plus 2 days and decitabine $20 \mathrm{mg} / \mathrm{m}^{2}$ endovenously for 5 days every 28 days have been widely used. Transfusion independence was achieved in most patients, with hematologic improvement (erythroid and thrombopoietic) in approximately $50 \%$ of cases, although neutrophil improvement was significant only for $34 \%$ of the patients. ${ }^{25}$

A recent study by the MDS Clinical Research Consortium used azacitidine or decitabine standard daily doses for 3 days every 28 days for a median of 9 cycles, with a response rate of $61 \%{ }^{27}$ Given the activity of HMAs in lower risk MDS, a phase III clinical trial with oral azacitidine (CC-486) is ongoing for lower risk MDS patients who are anemic and thrombocytopenic (ClinicalTrials.gov identifier, NCT01566695). However, the study is not strictly a clinical study for first-line therapy, because these patients could have previously received ESAs.

Immunosuppressive therapy has been underused in patients with MDS; however, lower risk MDS patients with pancytopenia have been treated with this modality with success. The reasons for the scarce use are diverse, including scanty evidence of activity, with studies including very small and heterogeneous groups of patients, the lack of characterization of the most active regimen, treatment toxicity, and the absence of parameters predictive of the response. However, a comparison of different immunosuppressive studies ${ }^{28}$ showed that alemtuzumab induced nearly $70 \%$ of responses. Quite surprisingly, cyclosporine, as a single drug, yielded more positive effects (56\% of response) than antithymocyte globulin (ATG) (horse ATG, 35\% response; rabbit ATG, 27\% response) and ATG plus cyclosporine (25\% and 30\% responses for horse and rabbit ATG, respectively). The results of this comparison should be interpreted with caution because of the heterogeneity of the treated patient populations and the differences in the selection of cases. In a recent study, the addition cyclosporine to ATG was significantly associated with a positive response, together with an early initiation of therapy. ${ }^{29}$ The factors that have been indicated as predictive of ATG response include hypocellular marrow, the absence of marrow blasts, a normal karyotype, HLA-DR 15 positivity, and STAT-3 mutant cytotoxic T cells. The National Institutes of Health developed a predictive algorithm for immunosuppressive therapy for MDS, according to which, for HLA-DR15-negative patients, the sum of the patient's age plus the number of months of RBC transfusion dependence must be $<58$. For HLA-DR15-positive patients, the sum of the patient's age plus the months of RBC transfusion dependence must be $<72 .{ }^{30}$

Lower risk MDS patients with severe thrombocytopenia constitute $<20 \%$ of cases. In this setting, only experimental agents are available for first-line therapy. The thrombopoietin mimetic agents romiplostim and eltrombopag have been approved for treatment of immune thrombocytopenic purpura and have been investigated as treatment of MDS. Romiplostim, although effective in increasing the platelet number and decreasing hemorrhagic episodes, also induced temporary augmentation of marrow blasts. Thus, its development as treatment of MDS has been discontinued. ${ }^{31}$ However, eltrombopag does not increase marrow blasts, and the results of a phase II study demonstrated that it induced a $47.5 \%$ platelet response compared with $9.7 \%$ of the placebo arm $(P<.001) .{ }^{32}$ The good tolerability of the drug and its oral formulation could support its clinical use as first-line treatment of patients with severe thrombocytopenic MDS.

\section{First-line Treatment of Higher Risk MDS}

The treatment of IPSS intermediate-2/high-risk, high-risk, and very high risk IPSS-R is more straightforward than that for lower risk MDS (Figure 2).

At present, the best therapy is still considered the one that includes an HMA (decitabine or azacitidine). ${ }^{33,34}$ It is clear that these agents have some peculiarity with respect to traditional chemotherapeutic agents in that the beneficial effects of HMAs are noted only after 2 to 4 cycles of therapy, ${ }^{35}$ and complete remission is quite rare. However, achievement of even only hematologic improvement has correlated with an advantage in overall survival. ${ }^{36}$ The activity of HMAs does not lead to eradication of the dysplastic clone, which was recently demonstrated by the persistence after decitabine therapy of clones carrying specific mutations, even in patients with a treatment response. ${ }^{37}$ Moreover, the HMA therapeutic effect is bound to be lost over time. ${ }^{38,39}$

Recently, reports have indicated a lack of survival advantage for MDS patients treated with azacitidine. ${ }^{40}$ These disappointing results can be ascribed to the short therapy duration, with a quite limited number of cycles; however, the findings reported did not allow a conclusion. Also, other reports have confirmed the prolonged overall survival with HMA therapy in real-life settings. ${ }^{41}$ At present, standard first-line therapy for patients with higher risk MDS should be azacitidine $75 \mathrm{mg} / \mathrm{m}^{2} /$ day for 7 days with a 28-day cycle or decitabine $20 \mathrm{mg} / \mathrm{m}^{2} /$ day for 5 days with a 28 -day cycle for $\geq 6$ cycles. $^{42}$

HMA therapy has many advantages, including that the age barrier to treatment has been overcome by the good tolerability of HMA, which has made therapy possible even for elderly frail patients. Also, hospitalization will not be necessary because of the low toxicity. However, treatment must continue until disease progression, the improvements will be somehow delayed, and, in the end, all patients will develop a relapse, clear indications that these agents are not the optimum treatment of MDS. ${ }^{42}$

Treatment interruptions invariably result in a loss of response, and patients with resistant or relapsed disease will survive $<6$ months. ${ }^{38,39}$ However, even when a response is obtained with decitabine or azacitidine, the duration has ranged from 6 to 26 months. In particular, MDS patients with a complex karyotype can achieve a response with HMA, but the response will last only for a few weeks. For these cases, in particular, upfront combinations of HMA with new experimental agents with different mechanisms of action could be a winning strategy and should be pursued as the first-line approach within clinical investigations.

HMAs act by DNA hypomethylation ${ }^{43}$; which (and whether) specific regions of DNA are the targets and whether this is the 
mechanism is the basis of the clinical activity remains to be clarified. We have demonstrated that differentially methylated regions in non-promoter regions differ between patients with chronic myelomonocytic leukemia with resistance to versus sensitivity to decitabine. ${ }^{44}$ The differences in the mode of action and activity between azacitidine and decitabine must also be investigated further. The response to HMAs has been approximately $60 \%$, with a significant population of patients with refractory disease. The cellular and individual mechanisms of resistance to HMAs are under evaluation. Also, markers of sensitivity such as TET2 mutations, ${ }^{45}$ overexpression of nucleotide metabolizing enzymes such as $U C K 1^{46}$ and of cytokines CXCL4 and CXCL7 ${ }^{44}$ must be consolidated. Novel schedules, doses, ${ }^{24}$ and combinations with other agents should be investigated to increase the efficacy of these drugs. For high-risk MDS patients with negative molecular predictive factors, new drugs should be used as front-line therapy, without waiting for HMT failure or relapse. Several attempts have already been made. ${ }^{47}$ A combination with histone deacetylase inhibitors has not been a winning strategy. ${ }^{48}$ The combination of azacitidine with entinostat yielded fewer trilineage responses than azacitidine alone. ${ }^{49}$ Also, no outstanding results were obtained with decitabine plus vorinostat. ${ }^{50}$ The comparison of azacitidine alone with azacitidine plus vorinostat and with azacitidine plus lenalidomide indicated a lack of survival advantage for both combinations. ${ }^{51}$

Numerous agents are being combined with azacitidine and decitabine in first-line treatment of high-risk MDS. Trials of combinations with chemotherapeutic agents such as idarubicin and trials of anti-FLT3 such as sorafenib are ongoing, and the results with agents such as pracinostat $^{52}$ and gemtuzumab ozogamicin ${ }^{53}$ are intriguing.

\section{Hematopoietic Stem Cell Transplantation}

Allogeneic stem cell transplantation remains the only curative option for both lower and higher risk MDS patients. It is almost never the first-line therapy, although it plays a part in childhood $\mathrm{MDS}^{54}$; however, it can be considered earlier in some specific cases. In addition, the toxicity and mortality of this therapy, although in constant decrease over the years, renders it less suitable for elderly patients and not suitable for patients with IPSS-R very low and lowrisk MDS. ${ }^{55}$

\section{Future Perspectives of Improvement for First-line Therapies}

The prognostic importance of somatic mutations in $\mathrm{MDS}^{56}$ will, in the near future, drive the therapeutic choices after the diagnosis. The development of target agents, such as IDH1 and IDH2 inhibitors, and inhibitors of spliceosome is indicating the route to personalized treatment of MDS.

In addition, clinical studies are characterizing subpopulations of patients particularly sensitive to new agents. The modified activin receptor IIB containing the molecule ACE-536 (luspatercept), when administered to lower risk MDS patients, induced transfusion independence in $40 \%$ of cases. However, the hematologic improvement rate was $65 \%$ for those with the refractory anemia with ring sideroblasts (RARS) World Health Organization subtype of MDS and was $\leq 84 \%$ if serum EPO $<500 \mathrm{U} / \mathrm{L}$ and the presence of ring sideroblasts were considered. ${ }^{57}$ Patients with RARS and an SF3B1 mutation achieved a response in $73.3 \%$ of cases. A randomized trial of luspatercept versus placebo is ongoing for transfusion-dependent RARS patients with a lost response to ESAs (irrespective of SF3B1 mutation; ClinicalTrials.gov identifier, NCT02631070). However, luspatercept could also be considered a good alternative for first-line therapy in SF3B1-mutated patients who are not eligible for ESAs.

Guadecitabine, previously known as SGI-110, is a dinucleotide of decitabine and deoxyguanosine that prolongs the in vivo exposure of decitabine by protecting it from deamination. The hypothesis is that prolonged decitabine in vivo exposure could translate into better efficacy and therefore, after the good results in HMA-relapsed cases, guadecitabine has also been evaluated as first-line treatment of MDS. $^{58}$

\section{References}

1. Greenberg P, Cox C, LeBeau MM, et al. International scoring system for evaluating prognosis in myelodysplastic syndromes. Blood 1997; 89:2079-88.

2. Greenberg PL, Tuechler H, Schanz J, et al. Revised International Prognostic Scoring System for myelodysplastic syndromes. Blood 2012; 120:2454-65.

3. Greenberg PL, Stone RM, Al-Kali A, et al. Myelodysplastic syndromes, version 2. 2017. NCCN clinical practice guidelines in oncology. J Natl Compr Cancer Netw 2017; 15:60-87.

4. Santini V. Treatment of low-risk myelodysplastic syndromes. Hematology Am Soc Hematol Educ Program 2016; 2016:462-9.

5. Nilsson-Ehle H, Birgegård G, Samuelsson J, et al. Quality of life, physical function and MRI T2* in elderly low-risk MDS patients treated to a haemoglobin level of $\geq 120 \mathrm{~g} / \mathrm{L}$ with darbepoetin alfa \pm filgrastim or erythrocyte transfusions. Eur J Haematol 2011; 87:244-52.

6. Temraz S, Santini V, Musallam K, et al. Iron overload and chelation therapy in myelodysplastic syndromes. Crit Rev Oncol Hematol 2014; 91:64-73.

7. Cheson BD, Greenberg PL, Bennett JM, et al. Clinical application and proposal for modification of the International Working Group (IWG) response criteria in myelodysplasia. Blood 2006; 108:419-25

8. Hellström-Lindberg E, Negrin R, Stein R, et al. Erythroid response to treatment with G-CSF plus erythropoietin for the anemia of patients with myelodysplastic syndromes: proposal for a predictive model. Br J Haematol 1997; 99:344-51.

9. Santini V, Schemenau J, Levis A, et al. Can the revised IPSS predict response to erythropoietic-stimulating agents in patients with classical IPSS low or intermediate-1 MDS? Blood 2013; 122:2286-8.

10. Kosmider O, Passet M, Santini V, et al. Are somatic mutations predictive of response to erythropoiesis stimulating agents in lower risk myelodysplastic syndromes? Haematologica 2016; 101:e280-3.

11. Park S, Kelaidi C, Sapena R, et al. Early introduction of ESA in low risk MDS patients may delay the need for RBC transfusion: a retrospective analysis on 112 patients. Leuk Res 2010; 34:1430-6.

12. Latagliata R, Oliva EN, Volpicelli P, et al. Twice-weekly high-dose rHuEpo for the treatment of anemia in patients with low-risk myelodysplastic syndromes. Acta Haematol 2008; 120:104-7.

13. Kelaidi C, Beyne-Rauzy O, Braun T, et al. High response rate and improved exercise capacity and quality of life with a new regimen of darbepoetin alfa with or without filgrastim in lower-risk myelodysplastic syndromes: a phase II study by the GFM. Ann Hematol 2013; 92:621-31.

14. Fenaux P, Santini V, Aloe-Spiriti A, et al. Randomized, double-blind, placebocontrolled, multicenter study evaluating Epoetin alfa versus placebo in anemic patients with IPSS low-Int1 risk MDS. Haematologica 2016; 101:s1, abstract P248.

15. Platzbecker U, Symeonidis A, Oliva EN, et al. Arcade (20090160): a phase 3 randomized placebo-controlled double-blind trial of darbepoetin alpha in the treatment of anemia in patients with low or intermediate-1 risk myelodysplastic syndromes. Haematologica 2016; 101:s1, abstract S128.

16. Jädersten M, Malcovati L, Dybedal I, et al. Erythropoietin and granulocyte-colony stimulating factor treatment associated with improved survival in myelodysplastic syndrome. J Clin Oncol 2008; 26:3607-13.

17. Smith SW, Sato M, Gore SD, et al. Erythropoiesis-stimulating agents are not associated with increased risk of thrombosis in patients with myelodysplastic syndromes. Haematologica 2012; 97:15-20.

18. Gyan E, Andrieu V, Sanna A, et al. Myelodysplastic syndromes with single neutropenia or thrombocytopenia are rarely refractory cytopenias with unilineage dysplasia by World Health Organization 2008 criteria and have favourable prognosis. Br J Haematol 2016; 175:975-9.

19. Santini V, Almeida A, Giagounidis A, et al. Efficacy and safety of lenalidomide versus placebo in RBC transfusion-dependent patients with IPSS low or intermediate-1-risk myelodysplastic syndromes without $\operatorname{del}(5 \mathrm{q})$ and unresponsive or 


\section{First-line Therapy for MDS}

refractory to erythropoiesis-stimulating agents: results from a randomized phase 3 study (CC-5013-MDS005). I Clin Oncol 2016; 34:2988-96.

20. Kelaidi C, Park S, Brechignac S, et al. Treatment of myelodysplastic syndromes with $5 \mathrm{q}$ deletion before the lenalidomide era: the GFM experience with EPO and thalidomide. Leuk Res 2008; 32:1049-53.

21. List AF, Bennett JM, Sekeres MA, et al. Extended survival and reduced risk of AML progression in erythroid-responsive lenalidomide-treated patients with lowerrisk del(5q) MDS. Leukemia 2014; 28:1033-40.

22. Saft L, Karimi M, Ghaderi M, et al. p53 protein expression independently predicts outcome in patients with lower-risk myelodysplastic syndromes with del(5q). Haematologica 2014; 99:1041-9.

23. Della Porta MG, Gallì A, Bacigalupo A, et al. Clinical effects of driver somatic mutations on the outcomes of patients with myelodysplastic syndromes treated with allogeneic hematopoietic stem-cell transplantation. J Clin Oncol 2016; 34 3627-37.

24. Welch JS, Petti AA, Miller CA, et al. p53 and decitabine in acute myeloid leukemia and myelodysplastic syndromes. N Engl J Med 2016; 375:2023-36.

25. Grinblatt DL, Sekeres MA, Komrokji RS, et al. Patients with myelodysplastic syndromes treated with azacitidine in clinical practice: the AVIDA registry. Leuk Lymphoma 2015; 56:887-95.

26. Garcia-Manero G, Jabbour E, Borthakur G, et al. Randomized open-label phase II study of decitabine in patients with low- or intermediate-risk myelodysplastic syndromes. J Clin Oncol 2013; 31:2548-53.

27. Short NJ, Garcia-Manero G, Montalban Bravo G. Low-dose hypomethylating agents (HMAs) are effective in patients (Pts) with low- or intermediate-1-risk myelodysplastic syndrome (MDS): a report on behalf of the MDS Clinical Research Consortium. Blood 2015; 126:94.

28. Parikh AR, Olnes MJ, Barrett AJ. Immunomodulatory treatment of myelodysplastic syndromes: antithymocyte globulin, cyclosporine, and alemtuzumab. Semin Hematol 2012; 49:304-11.

29. Komrokji RS, Mailloux AW, Chen DT, et al. A phase II multicenter rabbit anti-thymocyte globulin trial in patients with myelodysplastic syndromes identifying a novel model for response prediction. Haematologica 2014; 99: 1176-83.

30. Saunthararajah Y, Nakamura R, Wesley R, et al. A simple method to predict response to immunosuppressive therapy in patients with myelodysplastic syndrome. Blood 2003; 102:3025-7.

31. Giagounidis A, Mufti GJ, Fenaux P, et al. Results of a randomized, double-blind study of romiplostim versus placebo in patients with low/intermediate-1-risk myelodysplastic syndrome and thrombocytopenia. Cancer 2014; 120:1838-46.

32. Oliva EN, Alati C, Santini V, et al. Eltrombopag versus placebo for low-risk myelodysplastic syndromes with thrombocytopenia (EQoL-MDS): phase 1 results of a single-blind, randomised, controlled, phase 2 superiority trial. Lancet Hematol 2017; 4:e127-36.

33. Fenaux P, Mufti GJ, Hellstrom-Lindberg E, et al. Efficacy of azacitidine compared with that of conventional care regimens in the treatment of higher-risk myelodysplastic syndromes: a randomised, open-label, phase III study. Lancet Oncol 2009; 10:223-32.

34. Lübbert M, Suciu S, Baila L, et al. Low-dose decitabine versus best supportive care in elderly patients with intermediate- or high-risk myelodysplastic syndrome (MDS) ineligible for intensive chemotherapy: final results of the randomized phase III study of the European Organisation for Research and Treatment of Cancer Leukemia Group and the German MDS study group. J Clin Oncol 2011; 29:1987-96.

35. Santini V. Novel therapeutic strategies: hypomethylating agents and beyond. Hematology Am Soc Hematol Educ Program 2012; 2012:65-73.

36. Gore SD, Fenaux P, Santini V, et al. A multivariate analysis of the relationship between response and survival among patients with higher-risk myelodysplastic syndromes treated within azacitidine or conventional care regimens in the randomized AZA-001 trial. Haematologica 2013; 98:1067-72.

37. Merlevede J, Droin N, Qin T, et al. Mutation allele burden remains unchanged in chronic myelomonocytic leukaemia responding to hypomethylating agents. Nat Commun 2016; 7:10767.
38. Prébet T, Gore SD, Esterni B, et al. Outcome of high-risk myelodysplastic syndrome after azacitidine treatment failure. I Clin Oncol 2011; 29:3322-7.

39. Jabbour E, Garcia-Manero G, Batty N, et al. Outcome of patients with myelodysplastic syndrome after failure of decitabine therapy. Cancer 2010; 116:3830-4.

40. Bernal T, Martínez-Camblor P, Sánchez-García J, et al. Effectiveness of azacitidine in unselected high-risk myelodysplastic syndromes: results from the Spanish registry. Leukemia 2015; 29:1875-81.

41. Dinmohamed AG, van Norden Y, Visser O, et al. Effectiveness of azacitidine for the treatment of higher-risk myelodysplastic syndromes in daily practice: results from the Dutch population-based PHAROS MDS registry. Leukemia 2015; 29: 2449-51.

42. Santini V, Prebet T, Fenaux P, et al. Minimizing risk of hypomethylating agent failure in patients with higher-risk MDS and practical management recommendations. Leuk Res 2014; 38:1381-91.

43. Santini V, Kantarjian HM, Issa JP. Changes in DNA methylation in neoplasia: pathophysiology and therapeutic implications. Ann Intern Med 2001; 134:573-86.

44. Meldi K, Qin T, Buchi F, et al. Specific molecular signatures predict decitabine response in chronic myelomonocytic leukemia. J Clin Invest 2015; 125:185772.

45. Bejar R, Lord A, Stevenson K, Bar-Natan M, Pérez-Ladaga A, Zaneveld J. TET2 mutations predict response to hypomethylating agents in myelodysplastic syndrome patients. Blood 2014; 124:2705-12.

46. Valencia A, Masala E, Rossi A, et al. Expression of nucleoside-metabolizing enzymes in myelodysplastic syndromes and modulation of response to azacitidine. Leukemia 2014; 28:621-8.

47. Ornstein MC, Mukherjee S, Sekeres MA. More is better: combination therapies for myelodysplastic syndromes. Best Pract Res Clin Haematol 2015; 28:22-31.

48. Stahl M, Gore SD, Vey N, et al. Lost in translation? Ten years of development of histone deacetylase inhibitors in acute myeloid leukemia and myelodysplastic syndromes. Expert Opin Investig Drugs 2016; 25:307-17.

49. Prebet T, Sun Z, Figueroa ME, et al. Prolonged administration of azacitidine with or without entinostat for myelodysplastic syndrome and acute myeloid leukemia with myelodysplasia-related changes: results of the US Leukemia Intergroup trial E1905. J Clin Oncol 2014; 32:1242-8.

50. Kirschbaum M, Gojo I, Goldberg SL. A phase 1 clinical trial of vorinostat in combination with decitabine in patients with acute myeloid leukaemia or myelodysplastic syndrome. Br J Haematol 2014; 167:185-93.

51. DiNardo CD, Daver N, Jabbour E, et al. Sequential azacitidine and lenalidomide in patients with high-risk myelodysplastic syndromes and acute myeloid leukaemia: a single-arm, phase 1/2 study. Lancet Haematol 2015; 2:e12-20.

52. Garcia-Manero G, Montalban-Bravo G, Berdeja JG, et al. Phase 2, randomized, double-blind study of pracinostat in combination with azacitidine in patients with untreated, higher risk myelodysplastic syndromes. Cancer 2017; 123:994-1002.

53. Daver N, Kantarjian H, Ravandi F, et al. A phase II study of decitabine and gemtuzumab ozogamicin in newly diagnosed and relapsed acute myeloid leukemia and high-risk myelodysplastic syndrome. Leukemia 2016; 30:268-73.

54. Hasle H. Myelodysplastic and myeloproliferative disorders of childhood. Hematology Am Soc Hematol Educ Program 2016; 2016:598-604.

55. Saber W, Horowitz MM. Transplantation for myelodysplastic syndromes: who, when, and which conditioning regimens. Hematology Am Soc Hematol Educ Program 2016; 2016:478-84.

56. Bejar R, Papaemmanuil E, Haferlach T, et al. Somatic mutations in MDS patients are associated with clinical features and predict prognosis independent of the IPSS-R: analysis of combined datasets from the International Working Group for Prognosis in MDS-Molecular Committee. Blood 2015; 126:907.

57. Giagounidis A, Platzbecker U, Germing U, et al. Luspatercept treatment leads to long term increases in hemoglobin and reductions in transfusion burden in patients with low or intermediate-1 risk myelodysplastic syndromes (MDS): preliminary results from the phase 2 PACE-MDS. Blood 2015; 23, abstract 92.

58. Issa JP, Roboz G, Rizzieri D, et al. Safety and tolerability of guadecitabine (SGI-110) in patients with myelodysplastic syndrome and acute myeloid leukaemia: a multicentre, randomised, dose-escalation phase 1 study. Lancet Oncol $2015 ; 16: 1099-110$ 